Original Research Paper

\title{
Big Data Interoperability for E-Governance
}

\author{
${ }^{1}$ El Benany Med Mahmoud, ${ }^{1}$ El Beqqali Omar and ${ }^{2}$ Aris M. Ouksel \\ ${ }^{1}$ Department of Computer Science, Sidi Mohamed Ben Abdullah University, Fez, Morocco \\ ${ }^{2}$ Department of Computer Science, University of Illinois at Chicago, Chicago IL 60680, USA
}

\author{
Article history \\ Received: 30-04-2018 \\ Revised: 27-02-2019 \\ Accepted: 05-10-2019 \\ Corresponding Author: \\ El Benany Med Mahmoud \\ Department of Computer \\ Science, Sidi Mohamed Ben \\ Abdullah University, Fez, \\ Morocco \\ Email: medmhdbennannu@gmail.com
}

\begin{abstract}
The notion of governance is innate to any society in the word. The use of big-data allow governance as a competitive advantage to attract resources, with the emergence of the social web and exponential data generated accordingly. Such social web and exponential data can be analyzed to give the enlightened human beings about the good governance and maintain an intelligent global state of mega cities. The government agencies should collect data from heterogeneous sources. To improve the services and applications, these agencies offer the citizens and businesses, through big data analytics, which is a global opportunity to integrate a large number of public databases together, fraud detection and tax control activities. The impact of big data on these e-government applications strives to facilitate better governance by demonstrating its use in research and practice. Therefore, the current paper zeroes in on an approach based on the interoperability and integration of big data. In our approach, such data is gathered from several separate and heterogeneous government sources.
\end{abstract}

Keywords: E-Governance, Interoperability, Big Data, Analytics

\section{Introduction}

The notion of governance is innate to any society (Nussbaum, 2003). The evolution of governance models should pertinently adhere to the society and investigate the underlying opportunities, potentials, powers and capabilities of a novel, emerging internet technologies (Wang and Emurian, 2005). It is defined as a tool for perceiving and formulating decisions and their implementation that empower stakeholders and citizens. It is a mechanism of formation of decisions and their implementation, which constitutes applicability, usability and steering the resources of a country (Kumar and Sharma, 2016). Good governance has to establish consensus among the stakeholders with is the goal to improve the quality of life enjoyed by all citizens, Table 1 shows the different types of application governance. In resolution 2000/64, the United Nations Commission on Human Rights identified the key attributes of good governance as transparency, responsibility, accountability, participation, responsiveness to the needs of people (Bindé, 2005; Ferreira-Snyman and Ferreira, 2006). Thus, to elevate the process of good governance and in accordance with the identified attributes, electronic media have been associated with the governance for the sake of providing an accountable, transparent, fast and economical channel to broadcast information for the execution of government administration activities, introducing the term e-governance (Sharma, 2004).

Big data is one of these new technologies and architectures that can leverage the value of extremely large volumes of highly diverse data through real-time processing and analysis. Recently, it contained vast amounts of data that have been generated from various government sources, such as the internet, cloud systems and mobile devices (Hashem et al., 2015). E-governance is the use of modern information and communication technologies to improve the effectiveness, efficiency and service delivery to citizens and promote transparency (Behara et al., 2009). It provides the common framework and direction in the implementation of government policies for the following actors: Government to Government (G2G), Government to Business (G2B) and Government to Citizen (G2C). However, e-Governance may be unhelpful by suggesting, wrongly, that delivery of ICTs is an end in itself. The aim of e-governance is to provide all the government services efficiently and effectively to the public in a locality while maintaining the transparency and reliability at an economical price (Dawes, 2008). 
Table 1: Evolution of web governance (Kumar and Sharma, 2016)

\begin{tabular}{llll}
\hline Versions of web & Web 1.0 Read-only web & Web 2.0 Social web & Web 3.0 Semantic web \\
\hline Application & Electronic media (ICT) & Social media (Big Data) & Ontologies \\
Governance & $\begin{array}{l}\text { E-governance (online } \\
\text { information forms) }\end{array}$ & $\begin{array}{l}\text { S-governance (policy making } \\
\text { and monitoring) }\end{array}$ & $\begin{array}{l}\text { Sentiment intelligence } \\
\text { based governance }\end{array}$ \\
\hline
\end{tabular}

Its initiatives have demonstrated the marked impact on society. With the expansion in the use of e-governance services by its stakeholders, their viewpoints toward government are changing. The key benefits that facilitate this idea of progressive governance are good governance, trust and accountability, citizen's awareness and empowerment, citizen's welfare, democracy, nation's economic growth and 24/7 service model. In a narrow sense, e-governance consists of three main components: "(1) e-administration that improves government processes, (2) e-citizens and e-services that can connect citizens/businesses together and (3) e-society that can build interactions with and within the civil society." Addressing a government's technical issues makes egovernment "a subset of e-governance" that is defined as a process of reform in the way governments work, shares information and delivers services to external and internal clients. By implementing an e-governance initiative, government departments will streamline their processes, connect all the stakeholders, cut costs and improve the delivery of their services. Nevertheless, this implementation needs to ensure the communication and the interoperability between E-Government applications and the stakeholders (Evans and Yen, 2005; Janssen et al., 2014). From a scientific point of view, there are two levels of interoperability that have to be supported and exposed: The technological level that allows communication between distributed applications and the process level which supports the modeling of these processes. During the later years, the Web Services Framework has developed as a standard for the implementation of distributed applications (Perera et al., 2014; Singh and van Sinderen, 2016). It addresses both the technological and process level of integration and therefore is presented as a solution to the above described integration problem to collect, analyze and correlate information about how data was produced, what resources were involved and which tasks were executed? The following sections provide the technical details of the steps outlined above and cover the implementation architecture. The section below provides an overview of related works. The upcoming section describes the big data impact of e-governance of our approach, which is detailed in the description of some architectural components. Data collection, storage, analysis, visualization, data enrichment and data access processes are outlined. Towards the end of the paper, the proposed architecture of the solution to support egovernance big data analysis, monitoring are discussed. The paper concludes with future works and perspectives.

\section{Big Data Impact of E-Governance}

The benefits of using big data in e-government field, with the concept of the mega city, smart city, can consider the potential of adopting predictive analytics application to introducing smart grid technology in order to improve the efficient use of utility resources, services and applications. This offers citizens and businesses, through big data, an opportunity to integrate and interoperate a large number of public administrative databases. Thus, it is meant to expose an approach based on interoperability and integration of big data at different levels of egovernance and throughput data from multiple heterogeneous governments' sources (Dawes et al., 2016).

The review of company data provides insight into the chain of cause-and-effect relationships and facilitates understanding of the root causes of the resulting event. This therefore refers to the use of an event architecture, which represents an asynchronous communication model that relies on messages and disseminates information in a distributed environment. It improves traditional data integration techniques such as data replication, batch and the generation of business intelligence reports. The basic concepts related to this architecture include Business Event Management (BEM) and Complex Event Processing (CEP). These concepts are the solution of the problems of distributed data management. If it is not possible to create a single data access point, a business entity service that groups everything need to use events based on event processing, for example, this is a viable solution. This type of business technology is related to business process management (BPM) (Jeston, 2014), Business Activity Monitoring (BAM) (Patel et al., 2017), in addition with Business event management BEM and Complex Event Processor (CEP) (Izaguirre et al., 2011). Business activity monitoring and Complex Event Processor (CEP) are mostly focused on batch mode and real-time access to business performance indicators, including interactive, real-time dashboards, proactive alert generation and business provenance. Complex Event Processing (CEP) refers to the abstraction technique, where sets of low-level events are aggregated by means of event pattern (rule) descriptions, into abstracted 'highlevel' events that make sense to a business user while technology can add a historical perspective to BAM and enables root cause analysis and process discovery (Ebbers et al., 2013). Based on automatic discovery, the business provenance technology focuses on what actually has happened during the business process execution by collecting, correlating and analyzing operational data. 
The steps of this approach can be summarized as follows (Curbera et al., 2008):

1. Identifying data collection points that would generate data sealant to operational aspects of the business is the first step of the solution. This requires understanding the business context. Information and documentation about the business operations, process execution platforms and business models help determine the relevant probing points.

2. A generic data model that supports different aspects of the business must be in place in order to utilize the operational data.

3. Finally, the data collected must be correlated with and placed in the commercial context in order to have an integrated view.

The emergence of the social web and exponential data generated accordingly can be analyzed to give a human touch to governance. To move towards egovernance, we are exposing a new perspective in which e-governance is important to understanding the human component of big data. With the continued explosion of available data, government agencies have great difficulty using this data to improve their services to citizens and businesses. The collection, analysis and visualization of these data, by extracting useful information, are representative of big data challenges for better governance.

The impact of big data on governance facilitated by the application of big data exploitation is illustrated through the demonstration of its use in research and practice and allowing government organizations to become more flexible and efficient.

In this paper, we will extend our previous work by exposing a paradigm of big data interoperability based on the uses of Big Data analytics for better governance. Moreover, this paradigm presents the potentials and challenges of big data in public administration associated with data collecting, analyzing, visualizing and decision making as summarized in the lifecycle of actions of our approach depicted in the Fig. 1. This architecture is extended by a new social model of e-governance designed to be transparent and democratic, enabling visualization and reporting of information in real-time mode (Mohanty et al., 2013).

Our approach offers a full support for big data analytics (Russom, 2011; Zikopoulos et al., 2012) with a combination of products that allow the capabilities to collect data that can be done from any data source and analyze data by providing two dimensions to deal with in the data analysis. The first is the volume of data, and the second is the aspect correlation. When data is received from multiple sources, the correlation of information of interest within the same data source and between multiple data sources can be difficult.

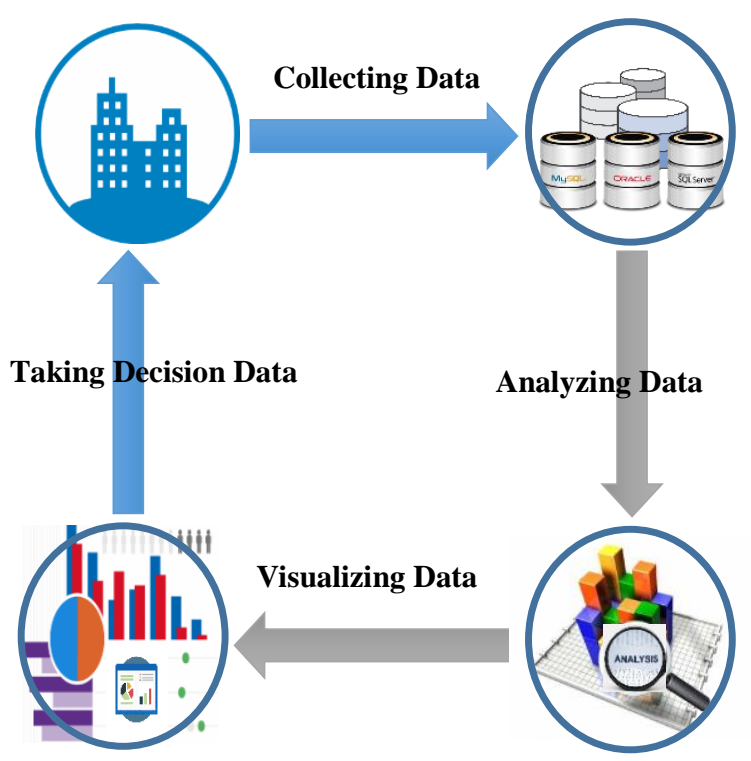

Fig. 1: Lifecycle of actions

The analysis has a temporal aspect. Some of them may occur in batch mode, as users who consume the results of the scan would be interested in them over time. However, other analyses, in order to be useful, must be done in real time and users will have to be informed instantly. In real-time analytics, users are interested in the results, as they become available, such as traffic monitoring, intelligent order routing, compliance monitoring and fraud detection. View analytics and detect Key Performance Indicators (KPIs) (Chan and Chan, 2004), take decision based on visualization results and KPI alerts (Stodder, 2013). This dimension, which is based on the visualization of the analyzed information, could be done in many ways. The most common and popular mechanism is to publish the summary of the analysis on the dashboards. Here, stakeholder interest will be responsible for monitoring KPIs. Stakeholders of a business can vary from employees of government agencies to partners that produce or consume services. Some examples of KPIs include the number of unique visitors to a website on a given day, the number of customers who go to a buyer in a sales funnel and the usage of data per user over a network. KPI monitoring can be performed both in real time and in batch mode. In the case of real time, there must be an alert template, and the dashboards may not work properly. With visualization, stakeholders and practitioners can make decisions based on what they see on the screens based on data analysis (Schmarzo, 2013). These decisions lead to action, such as changing process parameters and adjusting the process. Some of these actions could also be automated in order to enhance the performance of the proposed architecture. 
To summarize, our approach can identify the expectations based on the business perspectives in terms of solutions and products. The business perspective is able to make better decisions by operational monitoring, effectively communicate information by visualization and alerting mechanisms and finally offering new value proposition by insight driven product and services.

The solution perspective is based on the (1) - nonIntrusive integration that can polling and analyzing data from heterogeneous government data source and bushing results to existing flows with no interruption, (2) - intrusive integration by receiving data from multiple flow, systems and sources of data and (3) pushing the results to existing flows and systems. The product perspective can ensure the interoperability (1) in receiving data to analyze by multiple protocols and formats, pre-process before analyzing. (2) To communicate the results: Multiple protocols and formats (3) in data analysis by integrating with existing meta-data and models, extend with new analysis capabilities.

\section{Related Works}

The problem of using information and communication technologies is emerging to increase productivity and reduce the cost of labor. Thanks to this emergence, automation has been around since companies began using computers to automate their business processes to improve outstanding metrics such as quality of service and speed by drastically modifying existing needs in their business processes (Chambers and Dinsmore, 2014).

For all of this, current trends in collaborative governance, integrated governance and public interest approaches have stimulated interest in collaborative approaches for Big Data adoption in order to maximize IT business value by proposing predictive models to make efficiency and evidence based on business decisions. For all these reasons, current challenges in collaborative governance, integrated governance and public interest approaches have sparked interest in collaborative approaches to the adoption of Big Data to maximize the benefit of IT through the adoption of predictive models to make evidence-based, effective decisions. In the 1990s, the Business Process Reengineering (BPR) concept sparked tremendous discussions and greatly influenced the way people perceive their business, but will not be there for a long time. After some failures and abuses, the excitement around BPR failed. In the early 2000s, Business Process Management (BPM) began to be widely accepted by companies as a successor to BPR (Hyötyläinen, 2014). BPM also uses information and communication technologies to increase efficiency, productivity and manageability by taking a more formal but less radical approach (Kemsley, 2015).

This article contributes to the aspects of monitoring, analysis and optimization of the BPM discipline using Big Data technology. As big data continues to grow around the world, more and more governments are interested in related technologies to drive their development. In the face of exploding volume, variety and data velocity, 2018 is arguably the year of all records for Big Data and analytics. However, no matter what big data technologies are used for analytics tasks, business users need to be able to visualize and understand the results. Big Data technologies track endto-end business operations, based on specific instruments, to extract relevant information. Extraction of information from business processes is another technique that allows the analysis of these processes based on event logs (Chen et al., 2012; Van der Aalst and Weijters, 2004; Wu et al., 2014). However, our approach proposes an architecture based on the combination of SOA and EDA. This architecture will aim at ensuring interoperability in receiving, analyzing and visualizing data for e-government systems to ensure good governance in order to allow the flexibility of communication geographic government services independents of each other (Flood et al., 2016; Hamida et al., 2014; Marques-Lucena et al., 2016).

For e-governance, data across a stable network of service providers and consumers will be accessible through an Enterprise Service Bus (ESB) (Kadadi et al., 2014), which serves as a messaging backbone. It is a set of middleware services offering various types of capabilities integration. Such electronic services generally require the implementation of collaborative workflows of several organizations.

In this paper, we propose the use of the Big Data analytics approach for interoperability in e-government systems as a fundamental support mechanism for the interoperability of multiple workflows in geographically separated collaborative organizations.

\section{The Proposed Architecture}

Our proposed Big Data Interoperability Framework for e-governance (BDIF-eG), as shown in Fig. 2, is based on the big data analytics architecture. The BDIF$\mathrm{eG}$ is an extension of our previous work. It overcomes the limitations of the previous architectures in term of interoperability in receiving data, interoperability in communicating results and interoperability in data analysis. The workload, cost develops advanced interoperable features for better e-governance in central, local levels. The aims of interoperability are egovernance, e-administration, e-citizens, e-services and e-society all of which represent a real complexity of cross-organizational collaboration. 


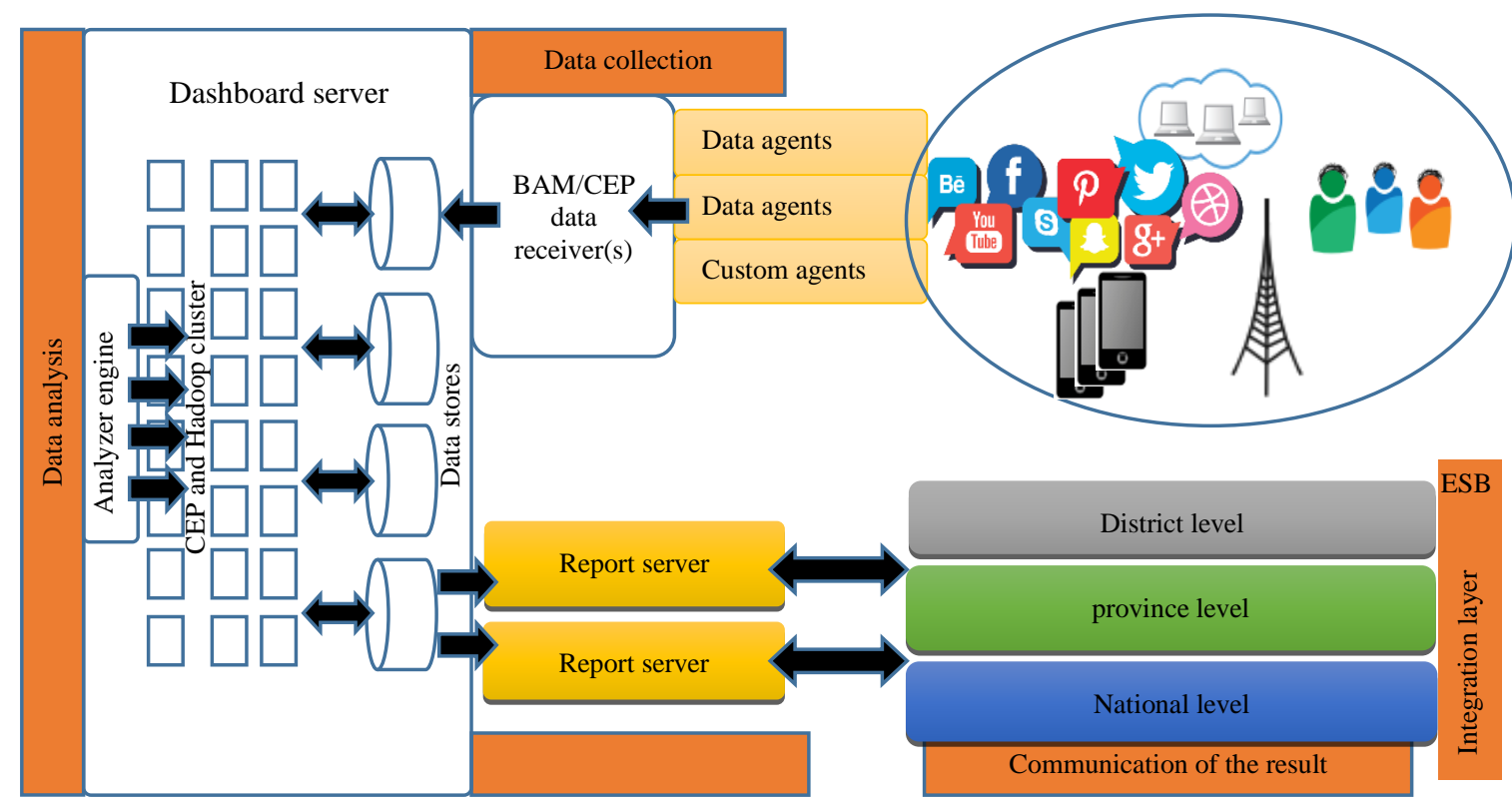

Fig. 2: Big Data Interoperability Framework for e-Governance (BDIF-eG)

These conclusions motivated the specification of a common framework for a big data interoperable platform that could be extended and refined to build concrete solutions for different e-governance scenarios. BDIF-eG is a comprehensive framework built upon our previous one to meet the needs of big data analytics to better e-governance in collect, analyze and visualize data stream. It comprises all the pieces required to achieve big data analytics from end to end. It can monitor both multiple data sources products and systems by using an agent-based architecture that has easy-to-use tools to gain access to the volumes of data. This architecture provides both real-time and batch mode tools, such as CEP (Moxey et al., 2010) and BAM (Wahl et al., 2010), to monitor streams of data; and has a complete toolset to build gadgets and dashboards for visualizing and tools to automate action taking and systems tuning, with the ability to incorporate human tasks to better make a decision:

- BDIF-eG contains BAM and CEP products with all the required tools and technologies such as analysis engines (e.g., Hadoop and Hive, Siddhi) and big data stores (e.g., Cassandra)

- BDIF-eG also allows integrating any existing platform that we have in governance mode. We can use the custom data agents to publish events and data we want can be received via custom data receivers

BDIF-eG is flexible and easy to adapt to our needs, then we can use any data schema we want and get the framework to capture and monitor those data. This is also true for data analysis and visualization.
For data analysis, we have the flexibility of defining the analysis logic to suit the needs. At the same time, BDIF-eG helps to schedule and deal with the time dimension of the analysis with ease. In case of visualizing, we have the flexibility of writing our own gadgets and laying them out the way we want on our dashboards.

Alternatively, the BDIF-eG also has provided for us to automatically generate gadgets with graphs and tables based on the summarized data streams.

The BDIF-eG is built from the ground up with a clean design with no acquisitions integrated. The key benefit is that the platform is tuned to fit our needs in the big data analytics space.

Our previous architecture contains an orchestration for e-government meta-services and e-government business process in trust government zone (national, province, district or eventually non-government agencies). The BDIF-eG integrates the previous one by adding new features based on analytics and using the Big data interoperability as an aspect to turn unstructured data into operational wisdom (El Benany and El Beqqali, 2016; 2015). The architecture is essentially cohesive and coherent, and it is developed to examine the emerging initiatives, validity and accuracy of the previous crossorganizational collaboration to meet those expectations.

\section{Data Collection}

For any incoming message that represents the data collected from any data source will be passed through the gateway to the analytic servers via the internet connection such as WIFI or LAN networks, and this data will use a higher level protocol, such as CoAp1or 
MQTT (Stockmann, 2016). The concept of "data agents" goes over the agents or customs agents that collect data. These data sources can be an Application Server, API Manager and Enterprise Service Bus (ESB), which are rebuilt by data agents that publish well-known data formats, such as statistics for service monitoring, usage monitoring and message mediation monitoring (Salisu, 2015). For custom data sources, we can implement customs agents with ease, using the APIs provided. The data agents publish streams of data into Business Activity Monitor (BAM) and Complex Event Processor (CEP):

- BAM captures these data streams into big data storages and then analyzes these stored data using the analyzing engine. Hence, the operation is in batch mode.

- CEP acts on incoming data streams in real-time or near real time mode (Kang and Han, 2008; VeraBaquero et al., 2016) but without storing data. The inbound processing occurs in real-time when data is received.

Data collection defines the data model, specifying the schema, picking a suitable protocol, transforming these data as needed and ingesting event.

\section{Data Analysis}

Both BAM and CEP generate results as streams. Stakeholders can act on these result streams. For BAM, they can store the results of the processed big data in RDBMS databases (as the volume of analyzing data is much smaller compared with original collected data), or they use another big data store (if the resulting space is large). Data analysis defines the selected type of analysis needed, configures the relevant analytics component (Chang, 2016; Vera-Baquero et al., 2013) and analyzes the received events.

\section{Data Visualizing}

The summary data is selected from several visualization tools such as User Engagement Server (UES) to build dashboards to monitor KPIs. In addition to KPI dashboards, CEP, which is more interesting, generates alerts for matching event detections, such as sending emails or SMS. However, CEP result streams can be captured by storage to help achieve delayed processing rather than real-time monitoring. The two approaches should be discussed: (1) The Communication of the results that defines the resulting schema, picking communication mode (Hashim, 2016) and communicate results. (2) The Integration Layer (Shrivastava and Pal, 2017) that has shown on this approach as a mechanism to improve interoperability. This approach should be easy to integrate and improve: a) Interoperability in data receiving that supporting multiple transport and formats and process incoming data before analysis

b) Interoperability in data analysis that assures integration with existing data store by looking up metadata store as event table, joining event tables with processing and updating the data store with processing. Integration with existing Models imports prediction models using Predictive Model Markup Language (PMML) data format, loading prediction models and uses these while processing. Importing Spark scripts for batch processing and extend additional analysis (Jun and Chung, 2016) by adding new functional capabilities as extensions to the underlying processing engine and adding the ability to run external scripts through integration extensions.

c) Interoperability in result communication by multiple transport protocols and data formats using alerting mode and storage capabilities

d) Interoperability in result visualization by integration with existing systems and dashboards. It allows the ability to build and deploy existing custom gadgets with existing dashboards and the ability of its integration with third-party visualization and reporting tools (Brohi et al., 2016; Lundquist et al., 2014).

\section{Conclusion}

The present paper investigates an architecture based on the big data analytics for e-governance. The core contribution was to provide a better e-government interoperability that allows data monitoring and an alert mechanism by using the combination of SOA and EDA. The most important advantage of our approach is the biggest data interoperability aspect that overcame the limitations reside in our previous work by collecting, analyzing and visualizing data from heterogeneous e-governance data source. The concept of data agents is the focus that can publish streams into Business Activity Monitor (BAM) and/or Complex Event Processor (CEP). The interoperability is assumed in receiving, communicating and analyzing data into a joint process at the national, provincial and district level on government agencies.

The perspective expounds on the shift from egovernance to s-governance by using an approach based on the sentiment analysis. The impact of Big Data on governance facilitated by application of sentiment mining was exposed by demonstrating its use in practice and research as well. At this point, the dynamic relationship between social media occurs for analyzing the content for sentiment mining in a tiresome task, as it requires thorough and extensive knowledge of the rules associated with NLP, like syntactic and semantic, explicit and implicit and regular 
and irregular language rules. Three main techniques will constitute our future research axes in the procedure of sentiment classification:

1) The machine learning approach that can be grouped into supervised and unsupervised learning methods.

2) The Lexicon-based approach tends that depends on the sentiment vocabulary that provides a collection of known and precompiled sentiment terms.

3) The hybrid approach that is the combination of both the above-mentioned methods and plays an important role in decision-making.

\section{Acknowledgment}

The author would like to thank all the people who have supported this work, as well as special thanks to Dr. Aris Ouksel, Professor of Information and Decision Sciences, from the University of Illinois at Chicago, USA.

\section{Author's Contributions}

El Benany Med Mahmoud: Designed the research plan, organized the study, contributed to the development, writing and formatting of the manuscript, proofreading and giving final approval of the manuscript to be submitted.

El Beqqali Omar and Aris M. Ouksel: Revision and supervision of the proposed model.

\section{Ethics}

This article is original and contains unpublished Material. The authors confirm that they have read and approved the manuscript and there is no conflict of interest. Also, the authors confirm that no ethical issues involved.

\section{References}

Behara, G.K., V.V. Varre and M. Rao, 2009. Service oriented architecture for e-governance. BPTrends.

Bindé, J., 2005. Towards knowledge societies. UNESCO world report.

Brohi, S.N., M.A. Bamiah and M.N. Brohi, 2016. Identifying and analyzing the transient and permanent barriers for big data. J. Eng. Sci. Technol., 11: 1793-1807.

Chambers, M. and T.W. Dinsmore, 2014. Advanced Analytics Methodologies: Driving Business Value with Analytics. 1st Edn., Pearson FT Press, ISBN-10: 0133498603, pp: 336.

Chan, A.P.C. and A.P.L. Chan, 2004. Key performance indicators for measuring construction success. Benchmarking: Int. J., 11: 203-221. DOI: $10.1108 / 14635770410532624$
Chang, J.F., 2016. Business Process Management Systems: Strategy and Implementation. 1st Edn., Auerbach Publications, ISBN-13: 9781420031362.

Chen, H., R.H. Chiang and V.C. Storey, 2012. Business intelligence and analytics: From big data to big impact. MIS Q. 36: 1165-1188.

DOI: $10.2307 / 41703503$

Curbera, F., Y. Doganata, A. Martens, N.K. Mukhi and A. Slominski, 2008. Business provenance-a technology to increase traceability of end-to-end operations. Proceedings of the OTM Confederated International Conferences "On the Move to Meaningful Internet Systems", Nov. 9-14, Springer, Mexico, pp: 100-119. DOI: 10.1007/978-3-540-88871-0_10

Dawes, S.S., 2008. The evolution and continuing challenges of e-governance. Public Admin. Rev., 68: S86-S102. DOI: 10.1111/j.1540-6210.2008.00981.X

Dawes, S.S., L. Vidiasova and O. Parkhimovich, 2016. Planning and designing open government data programs: An ecosystem approach. Government Inform. Q, 33: 15-27. DOI: 10.1016/j.giq.2016.01.003

Ebbers, M., A. Abdel-Gayed, V.B. Budhi, F. Dolot and V Kamat et al., 2013. Addressing Data Volume, Velocity and Variety with IBM InfoSphere streams V3.0. 1st Edn., IBM Redbooks. ISBN-10: 0738437808, pp: 320.

El Benany, M.M. and O. El Beqqali, 2016. Crossorganizational orchestrator for e-government interoperability. Proceedings of the IEEE/ACS 13th International Conference on Computer Systems and Applications, Nov. 29-Dec. 2, IEEE Xplore Press, Morocco, pp: 1-6.

DOI: $10.1109 / A I C C S A .2016 .7945750$

El Benany, M.M. and O. El Beqqali, 2015. SOA based egovernment interoperability. Proceedings of the IEEE/ACS 12th International Conference on Computer Systems and Applications, Nov. 17-20, IEEE Xplore Press, Morocco, pp: 1-2. DOI: $10.1109 / A I C C S A .2015 .7507251$

Evans, D. and D.C. Yen, 2005. E-government: An analysis for implementation: Framework for understanding cultural and social impact. Government Inform. Q, 22: 354-373. DOI: 10.1016/j.giq.2005.05.007

Ferreira-Snyman, M.P. and G.M. Ferreira, 2006. Global good governance and good global governance. South African Yearbook Int. Law, 31: 52-94. https://hdl.handle.net/10520/EJC100048

Flood, M.D., H.V. Jagadish and L. Raschid, 2016. Big data challenges and opportunities in financial stability monitoring. Financial Stability Rev., 20: 129-142.

Hamida, A.B., J. Lesbegueries, T. Morsellino, S. Zribi and J.P. Lorré, 2014. A Flexible Monitoring Infrastructure for a Cloud-Based ESB. In: Enterprise Interoperability VI, Mertins, K., F. Bénaben, R. Poler and J.P. Bourrières (Eds.), Springer, ISBN-10: 3319049488, pp: 261-269. 
Hashem, I.A.T., I. Yaqoob, N.B. Anuar, S. Mokhtar and A. Gani et al., 2015. The rise of big data on cloud computing: Review and open research issues. Inform. Syst., 47: 98-115. DOI: $10.1016 /$ j.is.2014.07.006

Hashim, A.Y.B., 2016. Big Data and Cloud Interoperability. In: Managing Big Data Integration in the Public Sector, Aggarwal, A. (Ed.), IGI Global, USA, ISBN-10: 1466696494 pp: 59-69.

Hyötyläinen, T., 2014. Steps to Improved Firm Performance with Business Process Management: Adding Business Value with Business Process Management and Its Systems. 1st Edn., Springer Gabler, ISBN-10: 3658074698, pp: 240.

Izaguirre, M.J.A.G., A. Lobov and J.L.M. Lastra, 2011. OPC-UA and DPWS interoperability for factory floor monitoring using complex event processing. Proceedings of the 9th IEEE International Conference on Industrial Informatics, Jul. 26-29, IEEE Xplore Press, Portugal, pp: 205-211. DOI: 10.1109/INDIN.2011.6034874

Janssen, M., E. Estevez and T. Janowski, 2014. Interoperability in big, open and linked dataorganizational maturity, capabilities and data portfolios. IEEE Comput., 47: 44-49.

DOI: 10.1109/MC.2014.290

Jeston, J., 2014. Business Process Management. 3rd Edn., Routledge, London, ISBN-13: 9781136172984. pp: 688.

Jun, C.N. and C.J. Chung, 2016. Big data analysis of local government 3.0: Focusing on Gyeongsangbukdo in Korea. Technol. Forecast. Soc. Change, 110: 3-12. DOI: 10.1016/j.techfore.2015.11.007

Kadadi, A., R. Agrawal, C. Nyamful and R. Atiq, 2014. Challenges of data integration and interoperability in big data. Proceedings of the IEEE International Conference on Big Data, Oct. 27-30, IEEE Xplore Press, USA, pp: 38-40.

DOI: 10.1109/BigData.2014.7004486

Kang, J.G. and K.H. Han, 2008. A business activity monitoring system supporting real-time business performance management. Proceedings of the 3rd International Conference on Convergence and Hybrid Information Technology, Nov. 11-13, IEEE Xplore Press, South Korea, pp: 473-478.

DOI: 10.1109/ICCIT.2008.224

Kemsley, S., 2015. Business Process Management and the Social Enterprise. Handbook on Business Process Management 1, vom Brocke, J. and M. Rosemann (Eds.), Springer, Berlin, pp: 463-474.

Kumar, A. and A. Sharma, 2016. Paradigm Shifts from E-governance to S-governance. In: The Human Element of Big Data: Issues, Analytics and Performance, Tomar, G. (Ed.), CRC Press, Boca Raton, ISBN-10: 1498754155.
Lundquist, D., K. Zhang and A. Ouksel, 2014. Ontologydriven cyber-security threat assessment based on sentiment analysis of network activity data. Proceedings of the International Conference on Cloud and Autonomic Computing, Sept. 8-12, IEEE Xplore Press, UK, pp: 5-14.

DOI: 10.1109/ICCAC.2014.42

Marques-Lucena, C., J. Ferreira, M. Sesana, K. Fisher and C. Agostinho, 2016. Process modelling approach for the liquid-sensing enterprise. Proceedings of the Interoperability for Enterprise Systems and Applications, (ESA' 16), At Guimarães, Portugal, Springer, Cham, pp: 211-223. DOI: 10.1007/978-3-319-30957-6_17.

Mohanty, S., Jagadeesh, M., Srivatsa, H., 2013. Big Data Imperatives Enterprise Big Data Warehouse, BI Implementations and Analytics. 1st Ed. Apress, New York, ISBN-13: 978-1-4302-4872-9.

Moxey, C., M. Edwards, O. Etzion, M. Ibrahim and S. Iyer et al., 2010. A conceptual model for event processing systems. IBM Redguide Publication.

Nussbaum, M., 2003. Gender and Governance: An Introduction. In: Essays on Gender and Governance, Nussbaum, M., A. Basu, Y. Tambiah and N. Gopal Jayal (Eds.), Human Development Resource Centre, UNDP, India, p: 1-19.

Patel, B., S. Roy, D. Bhattacharyya and T.H. Kim, 2017. Necessity of big data and analytics for good egovernance. Int. J. Grid Distributed Comput., 10: 11-20. DOI: 10.14257/ijgdc.2017.10.8.02

Perera, C., A. Zaslavsky, P. Christen and D. Georgakopoulos, 2014. Context aware computing for the internet of things: A survey. IEEE Commun. Surveys Tutorials, 16: 1408-0660. DOI: 10.1109/SURV.2013.042313.00197

Russom, P., 2011. Big data analytics. TDWI Best Pract. Rep. Fourth Quart.

Salisu, K., 2015. E-government adoption and framework for big data analytics. Proceedings of the 2nd Covenant University Conference on e-Governance in Nigeria, Jun. 10-12, Covenant University Canaanland, Ota Ogun State, Nigeria.

Schmarzo, B., 2013. Big Data: Understanding How Data Powers Big Business. John Wiley and Sons, New Jersey, ISBN-13: 9781118739570, pp: 240.

Sharma, P., 2004. E-governance. 1st Edn., APH Publishing Corporation, New Delhi, ISBN-10: 8176484792, pp: 340.

Shrivastava, S. and S.N. Pal, 2017. A big data analytics framework for enterprise service ecosystems in an egovernance scenario. Proceedings of the 10th International Conference on Theory and Practice of Electronic Governance, Mar. 7-9, ACM, India, pp: 5-11. DOI: $10.1145 / 3047273.3047274$ 
Singh, P.M. and M.J. van Sinderen, 2016. Big Data Interoperability Challenges for Logistics. In: Enterprise Interoperability in the Digitized and Networked Factory of the Future, Zelm, M., G. Doumeingts and J.P. Mendonca (Eds.), ISTE Press, UK, pp: 325-335.

Stockmann, D., 2016. Towards area-smart data science: Critical questions for working with big data from China. Leiden University, Netherlands.

Stodder, D., 2013. Data visualization and discovery for better business decisions. The Data Warehousing Institute, USA.

Van der Aalst, W.M. and A. Weijters, 2004. Process mining: A research agenda. Comput. Industry, 53: 231- 244. DOI: 10.1016/j.compind.2003.10.001

Vera-Baquero, A., R. Colomo-Palacios and O. Molloy, 2016. Real-time business activity monitoring and analysis of process performance on big-data domains. Telemat. Inform., 33: 793-807.

DOI: $10.1016 /$ j.tele.2015.12.005
Vera-Baquero, A., R. Colomo-Palacios and O. Molloy, 2013. Business process analytics using a big data approach. IT Profess., 15: 1391-8160.

DOI: 10.1109/MITP.2013.60

Wahl, A., A. Al-Moayed and B. Hollunder, 2010. An architecture to measure QoS compliance in SOA infrastructures. Proceedings of the 2nd International Conferences on Advanced Service Computing. Nov. 21-26, IRIA XPS Press, Lisbon, Portugal, pp: 27-33.

Wang, Y.D. and H.H. Emurian, 2005. An overview of online trust: Concepts, elements and implications. Comput. Human Behav., 21: 105-125. DOI: $10.1016 /$ j.chb.2003.11.008

Wu, X., X. Zhu, G.Q. Wu and W. Ding, 2014. Data mining with big data. IEEE Trans. Knowl. Data Eng., 26: 1393-4250. DOI: 10.1109/TKDE.2013.109

Zikopoulos, I., C. Paul, C. Eaton and P. Zikopoulos, 2012. Understanding Big Data: Analytics for Enterprise Class Hadoop and Streaming Data. 1st Ed. McGraw-Hill, New York, ISBN-13: 9780071790536, pp: 176. 